\title{
National and State-Specific Attitudes toward Smoke-Free Parks among U.S. Adults
}

\author{
Judy Kruger ${ }^{1, *}$, Amal Jama ${ }^{2}$, Michelle Kegler ${ }^{3}$, Kristy Marynak ${ }^{1}$ and Brian King ${ }^{1}$ \\ 1 Office on Smoking and Health, National Center for Chronic Disease Prevention and Health Promotion, \\ Centers for Disease Control and Prevention, Atlanta, GA 30306, USA; vhy4@cdc.gov (K.M.); \\ iyn3@cdc.gov (B.K.) \\ 2 DB Consulting Group, Atlanta, GA 30329, USA; vuj3@cdc.gov \\ 3 Emory University, Rollins School of Public Health, Atlanta, GA 30322, USA; mkegler@emory.edu \\ * Correspondence: jkruger@cdc.gov; Tel.: +1-011-404-639-2371
}

Academic Editors: Laura L. Jones and Amanda Farley

Received: 11 June 2016; Accepted: 26 August 2016; Published: 31 August 2016

\begin{abstract}
Outdoor places, such as parks, remain a source of secondhand smoke (SHS) exposure. We assessed attitudes toward smoke-free parks among U.S. adults. Data came from the 2009-2010 National Adult Tobacco Survey, a landline and cellular telephone survey of noninstitutionalized adults aged $\geq 18$ in the 50 U.S. states and D.C. Descriptive statistics and logistic regression were used to assess the prevalence and sociodemographic correlates of attitudes toward smoke-free parks, overall and by current tobacco use. Overall, 38.5\% of adults reported favorable attitudes toward complete smoke-free parks; prevalence ranged from 29.2\% in Kentucky to $48.2 \%$ in Maine. Prevalence of favorable attitudes toward smoke-free parks was higher among nonusers of tobacco $(44.6 \%)$ and noncombustible-only users (30.0\%) than any combustible users (21.3\%). The adjusted odds of having a favorable attitude were higher among: women; Hispanics and Black non-Hispanics, American Indian and Alaska Native non-Hispanics, and other non-Hispanics; those with an unspecified sexual orientation; and those with children aged $\leq 17$ in the household, relative to each characteristics respective referent group. Odds were lower among: any combustible tobacco and noncombustible-only tobacco users; adults aged 45-64; and those with some college or an undergraduate degree. Opportunities exist to educate the public about the benefits of smoke-free outdoor environments.
\end{abstract}

Keywords: parks; smoke-free; secondhand smoke; state; national; attitudes

\section{Introduction}

Exposure to secondhand smoke (SHS) has multiple significant negative effects on health across the lifespan, and the U.S. Surgeon General has concluded that there is no risk-free level of SHS exposure [1,2]. Each year in the U.S., SHS exposure causes more than 41,000 deaths among nonsmoking adults and 400 deaths among infants, and approximately $\$ 5.6$ billion in lost productivity [3]. Although population exposure to SHS has declined over the past two decades, many nonsmokers remain exposed to SHS in workplaces, homes, vehicles, and outdoor areas. SHS exposure in the U.S. population aged $\geq 3$ years declined from $52.5 \%$ during $1999-2000$ to $25.3 \%$ during 2011-2012; however, during 2011-2012, 58 million people were still exposed to SHS, including two in five children aged 3 to 11 years [4].

The World Health Organization Framework Convention on Tobacco Control recommends completely smoke-free environments to protect the public from SHS exposure in indoor worksites, public transport, indoor public places, and other indoor public places [5]. However, although there has been an increase in the adoption of smoke-free policies covering indoor public places, these policies do 
not protect people from SHS exposure in all public areas, including outdoor areas such as parks [6-9]. The implementation of policies prohibiting smoking in outdoor public places in the U.S., including parks, has increased in recent years [10-15]. As of October 2015, over 1100 municipalities in 46 states and the District of Columbia had enacted smoke-free park laws [16]. For example, New York City continues to generate success in denormalizing smoking in public places through the implementation of smoke-free laws, which has resulted in reduced smoking in parks and on beaches [17-19]. In addition, at the state and territory level, Oklahoma prohibits smoking on all indoor and outdoor state-owned land, Hawaii prohibits smoking in state parks, and Puerto Rico prohibits smoking in all parks [16].

Parks can be especially important places to adopt smoke-free policies because these setting are frequented by children, who are particularly susceptible to the adverse effects of SHS exposure, and who could view tobacco use as a socially acceptable behavior if exposed to it in public settings $[1,2,20]$. Evidence suggests that even low levels of exposure to SHS, such as that which could occur in outdoor settings, can have adverse health effects [2,21-24]. For example, a recent review examining the impact of SHS on outdoor air quality found that particulate matter with a diameter $\leq 2.5 \mu \mathrm{m}\left(\mathrm{PM}_{2.5}\right)$, which is a commonly used environmental marker for SHS, can accumulate in the lungs and is associated with numerous chronic respiratory and cardiovascular health complications [25]. Smoke-free policies could help reduce SHS exposure in parks and could lead to other positive outcomes, such as increased park attendance and revenue, increased park safety, and reduced environmental hazards [26-28]. Smoke-free parks could also help make tobacco use less socially acceptable [26-29], reduce fire hazards, reduce health risks to children and animals from ingestion of cigarette butts [14,30], and reduce pollution generated by discarded cigarette waste [31].

Although studies have assessed attitudes toward smoke-free policies in indoor areas and outdoor areas more generally [3], few studies have assessed attitudes toward smoke-free parks [17-19], and none have done so using national and state representative samples of U.S. adults. To address this gap, we assessed the national and state-specific prevalence of favorable attitudes toward smoke-free parks among U.S. adults, as well as sociodemographic correlates of favorable attitudes toward smoke-free parks overall and by current tobacco use at the national level.

\section{Materials and Methods}

\subsection{Data Source}

We used data from the 2009-2010 National Adult Tobacco Survey (NATS), a national landline and cellular telephone survey of noninstitutionalized civilian adults aged 18 years or older residing in the 50 U.S. states and the District of Columbia [32]. In brief, the 2009-2010 NATS used a stratified, multistage probability design to yield data representative at both the national and state levels. A complete description of the 2009-2010 NATS methodology is available elsewhere [33]. The NATS target population was non-institutionalized adults age 18 and older and respondents were selected from two sampling frames, one for landlines and one for cell phones. Between 20 October 2009 and 28 February 2010, a total of 118,581 interviews were conducted (landline $=110,634$ and cellular $=7947$ ). The overall response rate was $37.6 \%$ (landline $=40.4 \%$ and cellular $=24.9 \%$ ) [34]. State-specific response rates ranged from $28.2 \%$ in New Jersey to $49.3 \%$ in Vermont.

\subsection{Measures}

\subsubsection{Attitudes toward Smoke-Free Parks}

Respondents were asked about their attitudes toward SF parks using the question, "Should smoking at parks...'always be allowed', 'be allowed only at some times or in some places', or 'never be allowed'". Respondents who answered "never be allowed" were categorized as having a favorable attitude toward completely smoke-free parks. 


\subsubsection{Tobacco Use}

Current tobacco use status was determined by respondents' answers to questions on the current use of six tobacco products: cigarettes, cigars/cigarillos/little cigars, water pipes, pipes, chew/snuff/dip, and snus. Respondents were classified into three categories: (1) any combustible tobacco use; (2) noncombustible-only tobacco use; and (3) no tobacco use. "Any combustible tobacco use" was defined as a respondent who reported smoking at least 100 cigarettes during their lifetime and now smoked every day or some days and/or used cigars/cigarillos/little cigars, water pipes, or pipes on $\geq 1$ day during the past 30 days. "Noncombustible-only tobacco use" was defined as using chewing tobacco/snuff/dip or snus on $\geq 1$ day during the past 30 days, but did not use cigars/cigarillos/little cigars, water pipes, or pipes on $\geq 1$ day during the past 30 days, and reported currently using cigarettes "not at all". "No tobacco use" was defined as a respondent who did not ever use combustible (cigarettes, cigars/cigarillos/little cigars, water pipes, or pipes) or noncombustible (chewing tobacco/snuff/dip or snus) tobacco. It was not possible to further stratify the "no tobacco use" category by former and never use because of the threshold used to assess lifetime use, which was $\geq 1$ for most non-cigarette products. Additionally, e-cigarette use was not assessed in the 2009-2010 NATS, and thus, these products were not included in the definitions of tobacco use; however, the extent of use of these products was limited at the time of this survey [35], so the exclusion would not be expected to meaningfully impact this measure.

\subsubsection{Comprehensive Smoke-Free Law Coverage}

Comprehensive smoke-free law coverage was determined using the American Nonsmokers' Rights Foundation (ANRF) U.S. Tobacco Control Laws Database, which tracks U.S. municipal, county, and state laws relating to tobacco [36]. A comprehensive smoke-free law was defined as an ordinance or regulation that prohibits smoking in all indoor areas of non-hospitality workplaces, restaurants, and freestanding bars, including attached bars or separately ventilated rooms with no exemptions based on the number of employees. Laws included in this database are identified through systematic scanning of tobacco control publications, websites and e-mail discussion lists, biannual solicitation of information from tobacco control professionals, and partnerships. Identified tobacco control laws are coded by ANRF using standardized guidelines, and population protection estimates are calculated using data from the U.S. Census. For the purposes of this study, respondents were categorized according to the proportion of state residents covered by a state and/or local comprehensive smoke-free law as of 1 July 2010 [36]. Based on the distribution of the comprehensive smoke-free law coverage, the population fell into four categories: $100 \%$ (statewide comprehensive law); $20 \%-99 \% ; 1 \%-19 \%$; $0 \%$ (no local or statewide comprehensive laws) [37]. These categories were selected based on the population distribution of coverage across states to ensure that statistically reliable comparisons could be made across categories.

\subsubsection{Sociodemographic Characteristics}

Assessed sociodemographic characteristics included: sex (male or female), age (18-24, 25-44, $45-64$, or $\geq 65$ years), race/ethnicity (Hispanic and non-Hispanic white, black, Asian, American Indian/Alaska Native, Native Hawaiian/Pacific Islander, multi-race, or other); educational attainment (0-11 years (no diploma), Graduate Equivalency Degree (GED), high school graduate, some college (no degree), associate degree, undergraduate degree, or graduate degree), marital status (married/living with a partner, single/separated/divorced, or widowed), annual household income $(<\$ 20,000, \$ 20,000$ to $\$ 49,999, \$ 50,000$ to $\$ 99,999, \geq \$ 100,000$, or unspecified), U.S. Census region (Northeast, Midwest, South, or West), sexual orientation (heterosexual/straight, lesbian/gay/bisexual/transgender (LGBT), or unspecified); and if there were children aged $\leq 17$ years living in the household (yes or no). The aforementioned characteristics were chosen because they have previously been shown to influence the prevalence of tobacco use and attitudes toward tobacco control policies [38,39]. 


\subsection{Data Analysis}

Data were analyzed using SAS-Callable SUDAAN 10 (RTI International, Research Triangle Park, NC, USA) and weighted to adjust for the differential probabilities of selection and response. In order to create a data set with demographics matching the U.S. population, survey weights were generated based on sex, age, race/ethnicity, marital status, and educational attainment and telephone type, by state [33]. Because the distribution of cellular telephone respondents was less than the number of landline respondents in states, to prevent large variances and ensure adequate precision for state estimates, the national and state estimates were calculated differently. For national estimates, we included both cellular telephone and landline respondents. For state-specific estimates, we included cellular telephone respondents in states (California, Florida, Georgia, Illinois, Louisiana, New Jersey, New York, North Carolina, Ohio, Oklahoma, Pennsylvania, and Texas) that had a cellular telephone sample of 200 or more respondents [33].

Descriptive analyses, including point estimates and 95\% confidence intervals (CIs), were calculated overall and by current tobacco use status. In addition, multivariate logistic regression models were constructed with a favorable attitude toward completely smoke-free parks as the dependent variable. Covariates were sex, age group, race/ethnicity, educational attainment, marital status, annual household income, U.S. region, sexual orientation, whether children aged $\leq 17$ years were living in the household, and current tobacco use status. Regression models were constructed overall, as well as by each category of tobacco use (i.e., any combustible tobacco product, noncombustible-only tobacco use, no tobacco use), each of which adjusted for the same covariates as the overall model (excluding tobacco use).

\section{Results}

\subsection{National Prevalence of Attitudes toward Smoke-Free Parks}

Nationally, $18.9 \%$ of U.S. adults reported that smoking should "always be allowed at parks", $42.6 \%$ reported smoking should be "allowed only at some times or in some places", and 38.5\% reported smoking should "never be allowed" (Table 1). The prevalence of reporting that smoking should "never be allowed" at parks was higher among women (43.4\%) than men (33.6\%); among respondents with an unspecified sexual orientation (59.5\%) than heterosexual/straight (38.3\%) and LGBT respondents $(36.2 \%)$; and among respondents with children aged $\leq 17$ years living in the household $(43.2 \%)$ than those without children living in the household (35.3\%). The prevalence of reporting that smoking should "never be allowed" was lower among adults aged 45-64 (34.8\%) than other age groups $(18-24=39.7 \% ; 25-44=40.8 \% ; \geq 65=40.0 \%)$ and residents of states in the Midwest $(36.2 \%)$ than those in other regions (West $=39.6 \%$; Northeast $=39.2 \%$; South $=39.0 \%$ ). By tobacco use status, prevalence of reporting favorable attitudes toward smoke-free parks was higher among "no tobacco users" (44.6\%) and "noncombustible-only tobacco users" (30.0\%) than "any combustible tobacco users" $(21.3 \%)$. No difference was observed in the prevalence of reporting that smoking should "never be allowed" between those living in a state covered by a statewide comprehensive smoke-free law $(37.9 \%)$ and those living in a state not covered by a comprehensive state and/or local comprehensive smoke-free law $(37.6 \%)$.

\subsection{State Prevalence of Favorable Attitudes toward Smoke-Free Parks}

Prevalence of favorable attitudes toward completely smoke-free parks ranged from $29.2 \%$ in Kentucky to $48.2 \%$ in Maine (Table 2). Among "any combustible tobacco users", prevalence of reporting a favorable attitude ranged from $11.6 \%$ in Wyoming to $33.3 \%$ in Maryland. Among "noncombustible-only tobacco users", prevalence of reporting a favorable attitude ranged from $18.1 \%$ in Georgia to $46.2 \%$ in Mississippi. Among "no tobacco users", prevalence of reporting a favorable attitude towards smoke-free parks ranged from $34.9 \%$ in Colorado to $53.0 \%$ in Maine. 


\subsection{Correlates of Favorable Attitudes toward Smoke-Free Parks}

Table 3 shows the adjusted odds of favorability toward completely smoke-free parks, by sociodemographic characteristics and is relative to each characteristics respective referent group. Among all respondents, the odds of favorability were higher among: women (OR (odds ratio) $=1.4$ ); black non-Hispanics $(\mathrm{OR}=1.4)$; American Indian/Alaska Native non-Hispanics ( $\mathrm{OR}=1.3)$; other non-Hispanics $(\mathrm{OR}=1.6)$; Hispanics $(\mathrm{OR}=1.5)$; respondents who did not specify their sexual orientation ( $\mathrm{OR}=1.7)$; and adults living in households with children aged $\leq 17$ years $(\mathrm{OR}=1.3)$. The odds of favorability toward smoke-free parks were lower among: respondents aged 45-64 years $(\mathrm{OR}=0.8)$; and those with some college education $(\mathrm{OR}=0.8)$ or with an undergraduate degree $(\mathrm{OR}=0.8)$.

Among "any combustible tobacco users", odds of favorability towards smoke-free parks were higher among: women $(\mathrm{OR}=1.3)$; black non-Hispanics ( $\mathrm{OR}=1.5)$; Hispanics ( $\mathrm{OR}=1.6)$; and those with children $\leq 17$ living in the household $(\mathrm{OR}=1.5)$; and lower among those aged 45-64 years (OR $=0.6)$ (Table 3). Among "noncombustible-only tobacco users", odds were lower among Hispanics (OR = 0.1). Among "no tobacco users", odds were higher among: women $(\mathrm{OR}=1.4)$; black non-Hispanics $(\mathrm{OR}=1.4)$; Hispanics $(\mathrm{OR}=1.5)$; respondents who were married/living with a partner $(\mathrm{OR}=1.2)$; those who did not specify their sexual orientation ( $\mathrm{OR}=1.7)$; and adults with children aged $\leq 17$ years living in the household $(\mathrm{OR}=1.2)$. Among "no tobacco users", odds were lower among: those aged $45-64$ years $(\mathrm{OR}=0.8)$; non-Hispanics of multiple races $(\mathrm{OR}=0.7)$; and respondents with some college $(\mathrm{OR}=0.8)$ or an undergraduate degree $(\mathrm{OR}=0.8)$.

No significant association was observed between favorability toward smoke-free parks and population level coverage of state and/or local smoke-free laws in indoor public areas, either overall or by current tobacco use status (Table 3). 
Table 1. Attitudes toward smoking at parks, by selected characteristics-United States, National Adult Tobacco Survey (NATS) $2009-2010$.

\begin{tabular}{|c|c|c|c|c|}
\hline \multirow{3}{*}{ Characteristics } & \multicolumn{4}{|c|}{ Attitudes toward Smoking at Parks } \\
\hline & Unweighted Frequency & Should Always Be Allowed & Be Allowed only at Some Times or in Some Places & Never Be Allowed \\
\hline & $\mathbf{n}$ & $\%(95 \% \mathrm{CI})$ & $\%(95 \%$ CI $)$ & $\%(95 \%$ CI $)$ \\
\hline Overall & 97,978 & $18.9(18.4,19.4)$ & $42.6(41.9,43.3)$ & $38.5(37.9,39.2)$ \\
\hline \multicolumn{5}{|l|}{ Sex } \\
\hline Male & 38,908 & $23.5(22.7,24.4)$ & $42.9(41.8,43.9)$ & $33.6(32.6,34.6)$ \\
\hline Female & 59,070 & $14.3(13.8,14.9)$ & $42.3(41.5,43.1)$ & $43.4(42.5,44.2)$ \\
\hline \multicolumn{5}{|l|}{ Age (years) } \\
\hline $18-24$ & 4782 & $18.3(16.6,20.0)$ & $42.0(39.8,44.2)$ & $39.7(37.6,41.9)$ \\
\hline $25-44$ & 25,961 & $16.3(15.4,17.2)$ & $42.9(41.7,44.1)$ & $40.8(39.6,42.0)$ \\
\hline $45-64$ & 42,100 & $21.8(21.0,22.6)$ & $43.5(42.5,44.4)$ & $34.8(33.8,35.7)$ \\
\hline$\geq 65$ & 25,135 & $19.6(18.6,20.6)$ & $40.4(39.1,41.7)$ & $40.0(38.7,41.2)$ \\
\hline \multicolumn{5}{|l|}{ Race/ethnicity } \\
\hline White, non-Hispanic & 81,606 & $20.3(19.7,20.8)$ & $44.2(43.5,44.8)$ & $35.5(34.9,36.2)$ \\
\hline Black, non-Hispanic & 7272 & $17.9(16.2,19.5)$ & $37.9(35.8,40.0)$ & $44.2(42.1,46.4)$ \\
\hline Asian, non-Hispanic & 1641 & $6.4(4.6,8.2)$ & $50.0(44.1,55.9)$ & $43.6(38.0,49.3)$ \\
\hline AI/AN, non-Hispanic & 1523 & $24.4(19.7,29.2)$ & $38.6(33.1,44.1)$ & $37.0(31.5,42.5)$ \\
\hline NH/PI, non-Hispanic & 397 & $23.9(14.3,33.4)$ & $42.1(31.9,52.2)$ & $34.1(24.2,44.0)$ \\
\hline Multi-race, non-Hispanic & 1231 & $17.3(13.4,21.3)$ & $53.5(47.5,59.4)$ & $29.2(24.3,34.1)$ \\
\hline Other, non-Hispanic & 481 & $20.4(12.6,28.2)$ & $35.1(25.8,44.4)$ & $44.5(34.7,54.2)$ \\
\hline Hispanic & 3827 & $14.1(12.1,16.2)$ & $36.4(33.5,39.3)$ & $49.5(46.5,52.4)$ \\
\hline \multicolumn{5}{|l|}{ Education } \\
\hline $0-12$ years (no diploma) & 6700 & $22.0(20.0,24.0)$ & $36.3(33.7,38.8)$ & $41.8(39.2,44.3)$ \\
\hline Graduate Equivalency Degree & 1722 & $26.7(22.9,30.4)$ & $42.2(37.9,46.5)$ & $31.1(27.0,35.2)$ \\
\hline High school graduate & 21,162 & $21.2(20.1,22.2)$ & $40.8(39.5,42.1)$ & $38.0(36.8,39.3)$ \\
\hline Some college (no degree) & 15,827 & $19.6(18.4,20.8)$ & $44.5(43.1,46.0)$ & $35.9(34.5,37.3)$ \\
\hline Associate degree & 14,241 & $18.3(17.1,19.5)$ & $43.7(42.1,45.2)$ & $38.0(36.5,39.5)$ \\
\hline Undergraduate degree & 23,150 & $14.3(13.5,15.1)$ & $47.3(46.0,48.5)$ & $38.4(37.2,39.6)$ \\
\hline Graduate degree & 15,176 & $11.6(10.7,12.5)$ & $45.9(44.4,47.3)$ & $42.6(41.1,44.0)$ \\
\hline \multicolumn{5}{|l|}{ Marital status } \\
\hline Single/separated/divorced or widowed & 38,628 & $21.7(20.8,22.6)$ & $41.5(40.5,42.6)$ & $36.8(35.7,37.8)$ \\
\hline Married/living with a partner & 59,350 & $16.8(16.2,17.5)$ & $43.4(42.5,44.2)$ & $39.8(39.0,40.6)$ \\
\hline \multicolumn{5}{|l|}{ Annual household income } \\
\hline$<\$ 20,000$ & 11,068 & $23.6(21.9,25.2)$ & $37.2(35.2,39.2)$ & $39.3(37.2,41.3)$ \\
\hline$\$ 20,000-\$ 49,999$ & 31,669 & $20.3(19.4,21.3)$ & $41.6(40.4,42.8)$ & $38.1(36.9,39.2)$ \\
\hline$\$ 50,000-\$ 99,999$ & 33,395 & $17.5(16.7,18.3)$ & $45.0(43.8,46.1)$ & $37.5(36.4,38.6)$ \\
\hline$\geq \$ 100,000$ & 18,380 & $14.9(13.8,16.0)$ & $45.8(44.3,47.2)$ & $39.3(37.9,40.7)$ \\
\hline Ünspecified & 3466 & $17.9(15.1,20.8)$ & $38.1(34.7,41.6)$ & $43.9(40.3,47.6)$ \\
\hline
\end{tabular}


Table 1. Cont.

\begin{tabular}{|c|c|c|c|c|}
\hline \multirow{3}{*}{ Characteristics } & \multicolumn{4}{|c|}{ Attitudes toward Smoking at Parks } \\
\hline & Unweighted Frequency & Should Always Be Allowed & Be Allowed only at Some Times or in Some Places & Never Be Allowed \\
\hline & $\mathbf{n}$ & $\%(95 \%$ CI $)$ & $\%(95 \%$ CI $)$ & $\%(95 \% \mathrm{CI})$ \\
\hline \multicolumn{5}{|l|}{ U.S. region $^{\text {a }}$} \\
\hline West & 21,297 & $16.4(15.2,17.7)$ & $44.0(42.2,45.8)$ & $39.6(37.8,41.4)$ \\
\hline Northeast & 17,702 & $17.3(16.2,18.4)$ & $43.5(42.1,44.9)$ & $39.2(37.8,40.6)$ \\
\hline Midwest & 20,276 & $22.7(21.7,23.7)$ & $41.1(40.0,42.3)$ & $36.2(35.0,37.3)$ \\
\hline South & 38,703 & $18.9(18.0,19.7)$ & $42.2(41.1,43.2)$ & $39.0(37.9,40.0)$ \\
\hline \multicolumn{5}{|l|}{ Sexual orientation } \\
\hline Heterosexual/straight & 94,870 & $18.9(18.3,19.4)$ & $42.8(42.1,43.5)$ & $38.3(37.6,39.0)$ \\
\hline LGBT & 2186 & $23.4(19.6,27.2)$ & $40.5(36.2,44.7)$ & $36.2(31.7,40.6)$ \\
\hline Unspecified & 922 & $10.8(6.9,14.8)$ & $29.6(22.8,36.4)$ & $59.5(52.2,66.8)$ \\
\hline \multicolumn{5}{|l|}{ Children $\leq 17$ living in household } \\
\hline No & 67,301 & $21.6(20.9,22.3)$ & $43.1(42.3,43.9)$ & $35.3(34.5,36.0)$ \\
\hline Yes & 30,677 & $15.0(14.2,15.8)$ & $41.8(40.7,43.0)$ & $43.2(42.0,44.4)$ \\
\hline \multicolumn{5}{|c|}{ Proportion of state population covered by a comprehensive state and/or local smoke-free law ${ }^{c}$} \\
\hline $0 \%$ & 19,299 & $19.2(18.2,20.2)$ & $43.2(42.0,44.4)$ & $37.6(36.5,38.8)$ \\
\hline $1 \%-19 \%$ & 14,235 & $16.9(15.6,18.2)$ & $41.7(39.9,43.6)$ & $41.4(39.6,43.2)$ \\
\hline $20 \%-99 \%$ & 17,689 & $19.9(18.6,21.2)$ & $42.8(41.2,44.5)$ & $37.3(35.7,38.9)$ \\
\hline $100 \%$ & 44,587 & $19.4(18.7,20.2)$ & $42.7(41.7,43.6)$ & $37.9(37.0,38.9)$ \\
\hline \multicolumn{5}{|l|}{ Tobacco use $^{\text {b }}$} \\
\hline No tobacco use & 78,497 & $13.2(12.6,13.7)$ & $42.3(41.5,43.0)$ & $44.6(43.8,45.4)$ \\
\hline Noncombustible-only tobacco use & 1818 & $28.1(24.3,32.1)$ & $42.0(37.9,46.2)$ & $30.0(26.1,34.2)$ \\
\hline Any combustible tobacco use & 17,068 & $35.1(33.8,36.5)$ & $43.6(42.2,45.1)$ & $21.3(20.1,22.6)$ \\
\hline \multicolumn{5}{|c|}{$\begin{array}{l}\text { Abbreviations: AI/AN, American Indian/Alaska Native; CI, confidence interval; LGBT, lesbian, gay, bisexual, or transgender; NH/PI, Native Hawaiian/Pacific Islander. a West: } \\
\text { Alaska, Arizona, California, Colorado, Hawaii, Idaho, Montana, Nevada, New Mexico, Oregon, Utah, Washington, and Wyoming; Northeast: Connecticut, Maine, Massachusett,, } \\
\text { New Jersey, New Hampshire, New York, Pennsylvania, Rhode Island, and Vermont; Midwest: Illinois, Indiana, Iowa, Kansas, Michigan, Minnesota, Missouri, Nebraska, North Dakota, } \\
\text { Ohio, South Dakota, and Wisconsin; and South: Alabama, Arkansas, Delaware, District of Columbia, Florida, Georgia, Kentucky, Louisiana, Maryland, Mississippi, North Carolina, } \\
\text { Oklahoma, South Carolina, Tennessee, Texas, Virginia and West Virginia; b Any combustible tobacco use was defined as current use of at least } 100 \text { cigarettes during their lifetime, and } \\
\text { now smoked "every day" or "some days", and/or used cigars/cigarillos/little cigars, water pipes, or pipes on } \geq 1 \text { day during the past } 30 \text { days. Noncombustible-only tobacco use was } \\
\text { defined as use of chewing tobacco/snuff/dip or snus on } \geq 1 \text { day during the past } 30 \text { days, but did not use cigars/cigarillos/little cigars, water pipes, or pipes on } \geq 1 \text { day during the past } \\
30 \text { days, and reported currently using cigarettes "not at all". No tobacco use was defined as a respondent who did not currently use combustible (cigarettes, cigars/ cigarillos/little } \\
\text { cigars, water pipes, or pipes) or non-combustible (chewing tobacco/snuff/dip or snus) tobacco; ' Population coverage of smoke-free laws was based on the American Nonsmokers' } \\
\text { Rights Foundation U.S. Tobacco Control Laws Database and represents the percent of the population covered by a state and/or local comprehensive smoke-free laws as of } 1 \text { July } 2010 . \\
\text { A comprehensive smoke-free law is defined as one that prohibits smoking in all indoor areas of non-hospitality worksites, restaurants, and bars. }\end{array}$} \\
\hline
\end{tabular}


Table 2. National and state prevalence of favorable attitudes toward completely smoke-free parks, by current tobacco use status-United States, National Adult Tobacco Survey (NATS), 2009-2010.

\begin{tabular}{|c|c|c|c|c|}
\hline \multirow[b]{2}{*}{ State } & \multicolumn{4}{|c|}{ Favorable Attitude toward Completely Smoke-Free Parks a $(\%, 95 \% \mathrm{CI})$} \\
\hline & $\begin{array}{c}\text { Overall } \\
(n=94,629)\end{array}$ & $\begin{array}{l}\text { Any Combustible Tobacco Use }{ }^{b} \\
\qquad(n=16,193)\end{array}$ & $\begin{array}{l}\text { Noncombustible-Only Tobacco Use }{ }^{\mathrm{c}} \\
(n=1745)\end{array}$ & $\begin{array}{c}\text { No Tobacco Use }{ }^{\mathrm{d}} \\
\quad(n=76,691)\end{array}$ \\
\hline \multicolumn{5}{|c|}{$100 \%$ Comprehensive smoke-free Law coverage $\mathrm{e}$} \\
\hline Arizona & $40.1(35.2,44.9)$ & $18.4(7.7,29.1)$ & f & $44.6(39.3,49.9)$ \\
\hline Colorado & $30.5(27.1,34.0)$ & $16.3(8.6,24.0)$ & $\mathrm{f}$ & $34.9(31.2,38.5)$ \\
\hline Delaware & $39.5(35.6,43.3)$ & $20.0(12.6,27.4)$ & $\mathrm{f}$ & $45.7(41.5,49.9)$ \\
\hline District of Columbia & $33.1(28.6,37.5)$ & $14.7(7.7,21.6)$ & $\mathrm{f}$ & $39.1(34.2,44.0)$ \\
\hline Florida $\mathrm{g}$ & $40.0(36.9,43.1)$ & $21.5(15.8,27.3)$ & $\mathrm{f}$ & $45.542 .1,49.0)$ \\
\hline Hawaii & $33.2(29.5,37.0)$ & $12.2(5.8,18.7)$ & $\mathrm{f}$ & $39.8(35.6,44.0)$ \\
\hline Illinois $\mathrm{g}$ & $40.2(36.9,43.6)$ & $24.9(18.0,31.7)$ & $\mathrm{f}$ & $45.3(41.6,49.1)$ \\
\hline Iowa & $40.5(36.9,44.2)$ & $17.7(9.9,25.4)$ & $\mathrm{f}$ & $47.3(43.3,51.4)$ \\
\hline Kansas ${ }^{\mathrm{h}}$ & $34.1(30.5,37.8)$ & $18.8(9.5,28.1)$ & $\mathrm{f}$ & $38.8(34.8,42.8)$ \\
\hline Louisiana $\mathrm{g}$ & $37.8(35.6,40.0)$ & $20.3(16.5,24.2)$ & $31.4(20.3,42.4)$ & $46.1(43.5,48.6)$ \\
\hline Maine & $48.2(44.7,51.8)$ & $32.5(24.6,40.4)$ & $\mathrm{f}$ & $53.0(49.1,56.8)$ \\
\hline Maryland & $44.4(40.3,48.6)$ & $33.3(22.8,43.7)$ & $\mathrm{f}$ & $47.7(43.2,52.2)$ \\
\hline Massachusetts & $34.4(30.7,38.1)$ & $18.8(10.8,26.8)$ & $\mathrm{f}$ & $39.0(34.9,43.0)$ \\
\hline Michigan $^{\mathrm{h}}$ & $33.0(29.4,36.7)$ & $19.5(11.5,27.6)$ & $\mathrm{f}$ & $37.6(33.6,41.7)$ \\
\hline Minnesota & $37.5(33.9,41.1)$ & $17.4(9.8,25.0)$ & f & $41.5(37.6,45.5)$ \\
\hline Montana & $38.4(34.7,42.0)$ & $27.0(18.3,35.7)$ & $39.8(17.2,62.3)$ & $41.8(38.0,45.6)$ \\
\hline Nebraska & $37.0(33.4,40.5)$ & $16.5(10.1,22.9)$ & $\mathrm{f}$ & $43.5(39.4,47.6)$ \\
\hline Nevada & $29.2(25.7,32.7)$ & $16.0(9.2,22.7)$ & $\mathrm{f}$ & $35.4(31.2,39.6)$ \\
\hline New Jersey $g$ & $38.4(36.0,40.8)$ & $22.5(18.0,27.0)$ & $\mathrm{f}$ & $42.8(40.0,45.5)$ \\
\hline New Mexico & $39.0(34.6,43.4)$ & $23.5(13.8,33.2)$ & $\mathrm{f}$ & $45.0(40.2,49.9)$ \\
\hline New York $g$ & $39.7(36.5,42.8)$ & $22.9(16.4,29.3)$ & $\mathrm{f}$ & $44.2(40.6,47.8)$ \\
\hline Ohio $\mathrm{g}$ & $34.1(31.4,36.8)$ & $17.8(13.1,22.5)$ & $\mathrm{f}$ & $40.2(37.0,43.4)$ \\
\hline Oregon ${ }^{\mathrm{i}}$ & $33.2(29.5,36.9)$ & $20.0(10.7,29.2)$ & $\mathrm{f}$ & $36.4(32.5,40.3)$ \\
\hline Rhode Island & $44.8(40.8,48.9)$ & $31.7(23.2,40.2)$ & $\mathrm{f}$ & $50.0(45.6,54.4)$ \\
\hline Utah & $45.0(41.2,48.8)$ & $18.3(6.8,29.9)$ & $\mathrm{f}$ & $49.5(45.6,53.4)$ \\
\hline Vermont & $41.3(38.0,44.7)$ & $22.0(14.3,29.7)$ & $\mathrm{f}$ & $47.0(43.3,50.6)$ \\
\hline \multicolumn{5}{|c|}{$20 \%-99 \%$ Comprehensive smoke-free law coverage ${ }^{\mathrm{e}}$} \\
\hline Alaska & $34.2(30.7,37.6)$ & $17.9(11.3,24.5)$ & $\mathrm{f}$ & $41.0(37.1,44.9)$ \\
\hline Indiana & $40.8(37.2,44.5)$ & $24.7(16.5,32.8)$ & $\mathrm{f}$ & $47.4(43.4,51.3)$ \\
\hline Kentucky & $29.2(25.6,32.8)$ & $15.0(9.7,20.3)$ & $\mathrm{f}$ & $37.3(32.9,41.7)$ \\
\hline North Carolina $\mathrm{g}$ & $37.3(34.0,40.5)$ & $24.6(17.9,31.2)$ & $\mathrm{f}$ & $42.8(39.1,46.6)$ \\
\hline North Dakota $\mathrm{j}$ & $42.1(38.7,45.5)$ & $21.0(13.9,28.0)$ & c & $48.6(44.8,52.4)$ \\
\hline South Carolina & $41.9(39.2,44.6)$ & $25.5(20.6,30.5)$ & $\mathrm{f}$ & $48.3(45.2,51.4)$ \\
\hline Texas $\mathrm{g}$ & $39.1(36.0,42.2)$ & $22.0(16.2,27.7)$ & $\mathrm{f}$ & $45.2(41.6,48.8)$ \\
\hline Washington & $34.9(31.4,38.4)$ & $16.5(9.7,23.4)$ & $\mathrm{f}$ & $41.1(37.3,45.0)$ \\
\hline West Virginia & $36.6(32.9,40.2)$ & $17.2(10.5,23.8)$ & $34.8(18.3,51.3)$ & $44.5(40.2,48.8)$ \\
\hline
\end{tabular}


Table 2. Cont.

\begin{tabular}{|c|c|c|c|c|}
\hline \multirow[b]{2}{*}{ State } & \multicolumn{4}{|c|}{ Favorable Attitude toward Completely Smoke-Free Parks ${ }^{a}(\%, 95 \%$ CI) } \\
\hline & $\begin{array}{c}\text { Overall } \\
(n=94,629)\end{array}$ & $\begin{array}{l}\text { Any Combustible Tobacco Use }{ }^{b} \\
\qquad(n=16,193)\end{array}$ & $\begin{array}{c}\text { Noncombustible-Only Tobacco Use }{ }^{c} \\
(n=1745)\end{array}$ & $\begin{array}{l}\text { No Tobacco Use }{ }^{\mathrm{d}} \\
(n=76,691)\end{array}$ \\
\hline \multicolumn{5}{|c|}{$1 \%-19 \%$ Comprehensive smoke-free law coverage $\mathrm{e}$} \\
\hline Alabama & $38.7(35.0,42.5)$ & $22.1(15.6,28.6)$ & $\mathrm{f}$ & $46.1(41.7,50.4)$ \\
\hline Arkansas & $38.6(35.4,41.8)$ & $24.2(17.7,30.7)$ & $29.6(16.1,43.0)$ & $45.8(42.0,49.6)$ \\
\hline California ${ }^{g}$ & $44.5(41.3,47.7)$ & $25.3(18.8,31.9)$ & $\mathrm{f}$ & $49.5(45.9,53.2)$ \\
\hline Georgia $\mathrm{g}$ & $42.4(39.8,45.0)$ & $23.5(18.0,28.9)$ & $18.1(9.3,27.0)$ & $49.8(46.8,52.8)$ \\
\hline Mississippi & $42.1(37.7,46.5)$ & $31.9(21.5,42.3)$ & $46.2(29.4,63.0)$ & $46.3(41.4,51.3)$ \\
\hline Missouri & $32.0(28.6,35.4)$ & $13.1(7.9,18.4)$ & $\mathrm{f}$ & $39.3(35.4,43.3)$ \\
\hline Wisconsin ${ }^{j}$ & $34.6(31.0,38.1)$ & $16.0(6.6,25.5)$ & $\mathrm{f}$ & $39.5(35.7,43.3)$ \\
\hline Wyoming & $33.7(29.8,37.6)$ & $11.6(5.6,17.6)$ & $\mathrm{f}$ & $43.7(39.2,48.2)$ \\
\hline \multicolumn{5}{|c|}{$0 \%$ Comprehensive smoke-free law coverage $\mathrm{e}^{\mathrm{e}}$} \\
\hline Connecticut & $36.2(32.2,40.2)$ & $16.3(8.3,24.3)$ & f & $41.1(36.6,45.6)$ \\
\hline Idaho & $39.9(35.4,44.3)$ & $31.6(20.3,42.9)$ & $\mathrm{f}$ & $42.6(37.7,47.5)$ \\
\hline New Hampshire & $41.1(37.7,44.6)$ & $24.8(17.0,32.6)$ & $\mathrm{f}$ & $46.4(42.7,50.1)$ \\
\hline North Carolina $\mathrm{g}$ & $37.3(34.0,40.5)$ & $24.6(17.9,31.2)$ & f & $42.8(39.1,46.6)$ \\
\hline Oklahoma $\mathrm{g}$ & $37.7(35.6,39.9)$ & $21.4(17.8,24.9)$ & $31.3(19.5,43.1)$ & $46.4(43.8,49.0)$ \\
\hline Pennsylvania $\mathrm{g}$ & $40.0(37.6,42.3)$ & $22.4(17.9,27.0)$ & $\mathrm{f}$ & $46.2(43.6,48.9)$ \\
\hline South Dakota ${ }^{j}$ & $42.1(38.4,45.8)$ & $25.1(16.3,34.0)$ & $\mathrm{f}$ & $48.5(44.5,52.4)$ \\
\hline Tennessee & $38.3(34.5,42.1)$ & $25.5(16.8,34.2)$ & $\mathrm{f}$ & $44.7(40.4,49.0)$ \\
\hline Virginia & $37.6(34.0,41.3)$ & $16.1(10.4,21.8)$ & $\mathrm{f}$ & $44.9(40.7,49.1)$ \\
\hline National & $38.6(37.9,39.2)$ & $21.3(20.1,22.6)$ & $30.0(26.1,34.2)$ & $44.6(43.8,45.4)$ \\
\hline
\end{tabular}

Abbreviation: CI, confidence interval. a Favorable attitudes toward completely smoke-free parks was defined as a response of "never be allowed" to the question, "Should smoking at parks... always be allowed, be allowed only at some times or in some places, or never be allowed". For display purposes we have organized states based on the following categories: $100 \%$ (statewide comprehensive law); $20 \%-99 \% ; 1 \%-19 \% ; 0 \%$ (no local or statewide laws); ${ }^{b}$ Any combustible tobacco use was defined as current use of at least 100 cigarettes during their lifetime, and now smoked "every day" or "some days", and/or used cigars/cigarillos/little cigars, water pipes, or pipes on $>1$ day during the past 30 days; ${ }^{\mathrm{c}}$ Noncombustible-only tobacco use was defined as use of chewing tobacco/snuff/dip or snus on $>1$ day during the past 30 days, but did not use cigars/cigarillos/little cigars, water pipes, or pipes on $\geq 1$ day during the past 30 days, and reported currently using cigarettes "not at all"; ${ }^{\mathrm{d}}$ No tobacco use was defined as a respondent who did not currently use combustible (cigarettes,

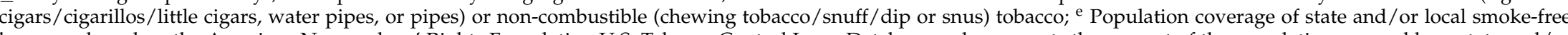
laws was based on the American Nonsmokers' Rights Foundation U.S. Tobacco Control Laws Database and represents the percent of the population covered by a state and/or local comprehensive smoke-free laws as of 1 July 2010. A comprehensive smoke-free law is defined as one that prohibits smoking in all indoor areas of non-hospitality worksites, restaurants, and bars; ${ }^{\mathrm{f}}$ Data not presented due to relative standard error $>30 \%$; $\mathrm{g}$ Calculated among landline and cellular telephone respondents. All other estimates calculated among landline respondents only; ${ }^{\mathrm{h}}$ Some states listed had implemented a comprehensive smoke-free law on the same day or around the same time as the ANR cut-off date of $1 \mathrm{July} 2010$ Michigan CSF law became effective on 1 May 2010; and Kansas CSF law became effective on 1 July 2010; ${ }^{i}$ Difference between current tobacco user and nontobacco user was significant (chi-square $p<0.05$ ) for all states except Oregon; ${ }^{j}$ Some states listed as not previously having a comprehensive smoke-free law now have a comprehensive smoke-free law after the ANR cut-off date of 1 July 2010.Wisconsin CSF law became effective on 5 July 2010; South Dakota CSF law became effective on 10 November 2010; and North Dakota CSF law became effective on 6 December 2010. 
Table 3. Adjusted odds of favorable attitudes toward completely smoke-free parks, by selected characteristics-United States, National Adult Tobacco Survey (NATS), 2009-2010.

\begin{tabular}{|c|c|c|c|c|}
\hline \multirow{3}{*}{ Characteristics } & \multicolumn{4}{|c|}{ Favorable Attitudes toward Completely Smoke-Free Parks a } \\
\hline & $\begin{array}{c}\text { Overall } \\
(n=94,629)\end{array}$ & $\begin{array}{l}\text { Any Combustible Tobacco Use }{ }^{\mathrm{b}} \\
\qquad(n=16,193)\end{array}$ & $\begin{array}{c}\text { Noncombustible-Only Tobacco Use }{ }^{\mathrm{c}} \\
(n=1745)\end{array}$ & $\begin{array}{l}\text { No Tobacco Use }{ }^{\mathrm{d}} \\
(n=76,691)\end{array}$ \\
\hline & AOR $(95 \% \mathrm{CI})^{\mathrm{e}}$ & AOR $(95 \% \mathrm{CI})^{\mathrm{e}}$ & $\operatorname{AOR}(95 \% \mathrm{CI})^{\mathrm{e}}$ & AOR $(95 \% \text { CI) })^{e}$ \\
\hline \multicolumn{5}{|l|}{ Sex } \\
\hline Male & 1.0 & 1.0 & 1.0 & 1.0 \\
\hline Female & $1.4(1.3,1.5)$ & $1.3(1.2,1.6)$ & $1.5(0.8,2.9)$ & $1.4(1.3,1.5)$ \\
\hline \multicolumn{5}{|l|}{ Age (years) } \\
\hline $18-24$ & 1.0 & 1.0 & 1.0 & 1.0 \\
\hline $25-44$ & $0.9(0.8,1.0)$ & $0.9(0.7,1.1)$ & $1.0(0.5,1.9)$ & $0.9(0.8,1.1)$ \\
\hline $45-64$ & $0.8(0.7,0.8)$ & $0.6(0.5,0.7)$ & $0.5(0.3,1.0)$ & $0.8(0.7,0.9)$ \\
\hline$\geq 65$ & $0.9(0.8,1.0)$ & $0.9(0.6,1.1)$ & $0.7(0.3,1.5)$ & $0.9(0.8,1.0)$ \\
\hline \multicolumn{5}{|l|}{ Race/Ethnicity } \\
\hline White, non-Hispanic & 1.0 & 1.0 & 1.0 & 1.0 \\
\hline Black, non-Hispanic & $1.4(1.3,1.5)$ & $1.5(1.2,2.0)$ & $2.2(1.0,5.1)$ & $1.4(1.2,1.5)$ \\
\hline Asian, non-Hispanic & $1.2(0.9,1.5)$ & $0.7(0.3,1.3)$ & $0.2(0.0,1.1)$ & $1.2(1.0,1.6)$ \\
\hline AI/AN, non-Hispanic & $1.3(1.0,1.7)$ & $1.2(0.8,1.9)$ & $1.3(0.4,4.0)$ & $1.4(1.0,1.9)$ \\
\hline NH/PI, non-Hispanic & $0.9(0.6,1.4)$ & $0.5(0.2,1.6)$ & $0.1(0.0,1.4)$ & $1.1(0.7,1.8)$ \\
\hline Multi-race, non-Hispanic & $0.8(0.6,1.0)$ & $1.0(0.6,1.7)$ & $0.4(0.1,2.1)$ & $0.7(0.5,0.9)$ \\
\hline Other, non-Hispanic & $1.6(1.1,2.4)$ & $1.7(0.7,4.0)$ & $\mathrm{f}$ & $1.5(1.0,2.3)$ \\
\hline Hispanic & $1.5(1.4,1.7)$ & $1.6(1.2,2.2)$ & $0.1(0.0,0.6)$ & $1.5(1.3,1.7)$ \\
\hline \multicolumn{5}{|l|}{ Education } \\
\hline 0-12 years (no diploma) & 1.0 & 1.0 & 1.0 & 1.0 \\
\hline Graduate Equivalency Degree & $0.8(0.6,1.0)$ & $0.9(0.6,1.3)$ & $0.7(0.2,3.0)$ & $0.8(0.6,1.0)$ \\
\hline High school graduate & $0.9(0.8,1.0)$ & $1.0(0.8,1.2)$ & $1.1(0.6,2.0)$ & $0.9(0.7,1.0)$ \\
\hline Some college (no degree) & $0.8(0.7,0.9)$ & $0.9(0.7,1.1)$ & $1.0(0.5,2.1)$ & $0.8(0.6,0.9)$ \\
\hline Associate degree & $0.9(0.8,1.0)$ & $0.9(0.7,1.1)$ & $0.7(0.3,1.5)$ & $0.9(0.7,1.0)$ \\
\hline Undergraduate degree & $0.8(0.7,0.9)$ & $1.0(0.8,1.3)$ & $0.6(0.3,1.3)$ & $0.8(0.7,0.9)$ \\
\hline Graduate degree & $0.9(0.8,1.1)$ & $0.9(0.7,1.3)$ & $0.9(0.3,2.3)$ & $0.9(0.8,1.1)$ \\
\hline \multicolumn{5}{|l|}{ Marital status } \\
\hline Single/separated/divorced or widowed & 1.0 & 1.0 & 1.0 & 1.0 \\
\hline Married/living with a partner & $1.1(1.0,1.2)$ & $1.0(0.8,1.1)$ & $1.1(0.7,1.7)$ & $1.2(1.1,1.3)$ \\
\hline \multicolumn{5}{|l|}{ Annual household income } \\
\hline$<\$ 20,000$ & 1.0 & 1.0 & 1.0 & 1.0 \\
\hline$\$ 20,000-\$ 49,999$ & $0.9(0.8,1.0)$ & $0.9(0.7,1.1)$ & $0.8(0.4,1.6)$ & $0.9(0.8,1.1)$ \\
\hline$\$ 50,000-\$ 99,999$ & $0.9(0.8,1.0)$ & $1.0(0.7,1.2)$ & $1.1(0.5,2.2)$ & $0.9(0.8,1.0)$ \\
\hline$\geq \$ 100,000$ & $0.9(0.8,1.1)$ & $1.1(0.8,1.5)$ & $1.0(0.4,2.2)$ & $0.9(0.8,1.0)$ \\
\hline Unspecified & $1.0(0.9,1.2)$ & $0.8(0.5,1.2)$ & $0.8(0.3,2.4)$ & $1.1(0.9,1.3)$ \\
\hline
\end{tabular}


Table 3. Cont.

\begin{tabular}{|c|c|c|c|c|}
\hline \multirow{3}{*}{ Characteristics } & \multicolumn{4}{|c|}{ Favorable Attitudes toward Completely Smoke-Free Parks ${ }^{\text {a }}$} \\
\hline & $\begin{array}{c}\text { Overall } \\
(n=94,629)\end{array}$ & $\begin{array}{c}\text { Any Combustible Tobacco Use }{ }^{b} \\
(n=16,193)\end{array}$ & $\begin{array}{c}\text { Noncombustible-Only Tobacco Use }{ }^{\mathrm{c}} \\
(n=1745)\end{array}$ & $\begin{array}{c}\text { No Tobacco Use }{ }^{\mathrm{d}} \\
(n=76,691)\end{array}$ \\
\hline & AOR $(95 \% \mathrm{CI})^{\mathrm{e}}$ & AOR $(95 \% \mathrm{CI})^{\mathrm{e}}$ & AOR $(95 \% \mathrm{CI})^{\mathrm{e}}$ & AOR $(95 \% \mathrm{CI})^{\mathrm{e}}$ \\
\hline \multicolumn{5}{|l|}{ U.S. region $\mathrm{g}$} \\
\hline West & 1.0 & 1.0 & 1.0 & 1.0 \\
\hline Northeast & $1.1(1.0,1.2)$ & $1.1(0.9,1.4)$ & $1.4(0.7,2.9)$ & $1.1(1.0,1.2)$ \\
\hline Midwest & $1.0(0.9,1.1)$ & $0.9(0.7,1.2)$ & $1.8(1.0,3.1)$ & $1.0(0.9,1.1)$ \\
\hline South & $1.1(1.0,1.2)$ & $1.1(0.9,1.3)$ & $1.4(0.8,2.5)$ & $1.1(1.0,1.2)$ \\
\hline \multicolumn{5}{|l|}{ Sexual orientation } \\
\hline Heterosexual/straight & 1.0 & 1.0 & 1.0 & 1.0 \\
\hline LGBT & $1.0(0.8,1.2)$ & $0.9(0.6,1.4)$ & $1.4(0.3,6.6)$ & $1.1(0.8,1.3)$ \\
\hline Unspecified & $1.7(1.3,2.4)$ & $2.2(0.9,5.0)$ & $0.5(0.1,2.1)$ & $1.7(1.2,2.4)$ \\
\hline \multicolumn{5}{|l|}{ Children $\leq 17$ living in household } \\
\hline No & 1.0 & 1.0 & 1.0 & 1.0 \\
\hline Yes & $1.3(1.2,1.4)$ & $1.5(1.3,1.8)$ & $0.8(0.5,1.2)$ & $1.2(1.1,1.3)$ \\
\hline \multicolumn{5}{|c|}{ Proportion of state population covered by a comprehensive state and/or local smoke-free law ${ }^{\mathrm{h}}$} \\
\hline $0 \%$ & 1.0 & 1.0 & 1.0 & 1.0 \\
\hline $1 \%-19 \%$ & $1.0(0.9,1.1)$ & $1.0(0.8,1.2)$ & $1.4(0.8,2.5)$ & $1.0(0.9,1.1)$ \\
\hline $20 \%-99 \%$ & $1.1(1.0,1.2)$ & $1.0(0.8,1.3)$ & $1.1(0.7,2.0)$ & $1.1(1.0,1.2)$ \\
\hline $100 \%$ & $1.0(0.9,1.1)$ & $1.0(0.8,1.2)$ & $1.4(0.8,2.5)$ & $1.0(0.9,1.1)$ \\
\hline \multicolumn{5}{|l|}{ Tobacco use $^{i}$} \\
\hline No tobacco use & 1.0 & - & - & - \\
\hline Noncombustible-only tobacco use & $0.6(0.5,0.6)$ & - & - & - \\
\hline Any combustible tobacco use & $0.3(0.3,0.4)$ & - & - & - \\
\hline
\end{tabular}

Abbreviations: AOR, adjusted odds ratio; AI/AN, American Indian/Alaska Native; CI, confidence interval; LGBT, Lesbian, Gay, Bisexual, or Transgender; NH/PI, Native Hawaiian/Pacific Islander. " Favorable attitudes toward completely smoke-free parks was defined as a response of "never be allowed" to the question, "Should smoking at parks... always be allowed, be allowed only at some times or in some places, or never be allowed"; ${ }^{\mathrm{b}}$ Any combustible tobacco use was defined as current use of at least 100 cigarettes during their lifetime, and now smoked "every day" or "some days", and/or used cigars/cigarillos/little cigars, water pipes, or pipes on $>1$ day during the past 30 days; ${ }^{c}$ Noncombustible-only tobacco use was defined as use of chewing tobacco/snuff/dip or snus on $\geq 1$ day during the past 30 days, but did not use cigars/cigarillos/little cigars, water pipes, or pipes on $\geq 1$ day during the past 30 days, and reported currently using cigarettes "not at all"; ${ }^{\mathrm{d}}$ No tobacco use was defined as a respondent who did not currently use combustible (cigarettes, cigars/cigarillos/little cigars, water pipes, or pipes) or non-combustible (chewing tobacco/snuff/dip or snus) tobacco; ${ }^{\mathrm{e}}$ Odds ratios adjusted for all other covariates listed in the table. Statistically significant odds ratios noted in bold; ${ }^{f}$ Data not presented due to relative standard error $\geq 30 \%$; $\mathrm{g}$ West: Alaska, Arizona, California, Colorado, Hawaii, Idaho, Montana, Nevada, New Mexico, Oregon, Utah, Washington, and Wyoming; Northeast: Connecticut, Maine, Massachusetts, New Jersey, New Hampshire, New York, Pennsylvania, Rhode Island, and Vermont; Midzest: a Delaware, Distictof 'Proportion of the state population covered by a state and/or local comprehensive smoke-free law was based on the American Nonsmokers' Rights Foundation U.S. Tobacco Control Laws Database, and represents the percent of the population covered by state and/or local comprehensive smoke-free laws as of 1 July 2010 . A comprehensive smoke-free law is defined as one that prohibits smoking in all indoor areas of non-hospitality worksites, restaurants, and bars; ${ }^{i}$ Overall favorable attitude towards completely smoke-free parks was defined as a response of yes to the question "Should smoking at parks never be allowed?". 


\section{Discussion}

This study found that about two in five U.S. adults had a favorable attitude toward smoke-free parks during 2009-2010. However, marked variations in favorable attitudes toward smoking restrictions in parks were observed across sociodemographic groups, current tobacco use status, and states. Although awareness of the harms of SHS exposure and the benefits of smoke-free public places have increased over time [39,40], these findings suggest that there are opportunities to further raise public awareness about the public health importance and benefits of smoke-free parks. The benefits of adopting smoke-free policies in outdoor areas such as parks extend beyond the protection of children and nonsmokers from SHS exposure and can also include reductions in litter, fire risks, and the social acceptability of tobacco use [14,30,31]. A better understanding of the general public's attitudes toward smoke-free parks may inform strategies to adopt and enforce such policies in this setting [21,26].

Irrespective of current tobacco use status, women, Hispanics, non-Hispanic racial/ethnic minorities, and adults with children aged $\leq 17$ years in the household were more likely to favor smoke-free parks than were men, white non-Hispanics, and adults without children aged $\leq 17$ years in the household, which is consistent with other studies [17,27]. Additionally consistent with other studies of cigarette smokers' attitudes toward indoor smoke-free policies, as well as surveys of public favorability toward tobacco-related policies [8,38], "any combustible tobacco users" had lower levels of favorability toward smoke-free park policies than "noncombustible-only tobacco users" or "no tobacco users". However, given that only $45 \%$ of nonusers of tobacco reported favorability toward completely smoke-free policies in parks, efforts to promote awareness of the benefits of smoke-free policies in this setting are still warranted.

No variation was observed in the odds of favorability toward smoke-free policies by the proportion of the population covered by state and/or local comprehensive smoke-free laws in indoor public areas. This finding may be the result of broader adoption of smoke-free park policies in jurisdictions across the country, irrespective of whether the jurisdiction has a comprehensive state and/or local smoke-free law. Smoke-free park policies are rapidly growing in popularity; 1101 counties contained at least one jurisdiction with a smoke-free park policy in 2015, compared to 432 counties in 2010 (ANRF database unpublished; http:/ /no-smoke.org) [16]. Favorable attitudes towards smoke-free policies have been found to increase after the implementation of smoke-free policies, while concern about the harms of secondhand smoke have been found to decrease [39]. Therefore, support for such policies is expected to continue to increase in the future, as smoke-free policies in indoor and outdoor public places, as well as attitudes regarding the social unacceptability of smoking, continue to proliferate [38,40-43]. However, given the greater population-level protection afforded by smoke-free policies in worksites and public places, smoke-free park policies are best suited for consideration following the implementation of comprehensive smoke-free policies in all indoor public places and worksites, including restaurants and bars.

Although most respondents reported attitudes supporting some level of smoking restrictions in parks, $43 \%$ of respondents reported that smoking should be "allowed only at some times or in some places". Future qualitative research is warranted to explore attitudes toward these partial restrictions in depth. Specific factors to explore could include whether attitudes vary depending on the park feature (e.g., playground, beach, picnic areas, trails), presence of children, or number or density of visitors. Further exploration of the factors motivating current levels of support may also be helpful, including protection of children, reduced fire risk, and concerns about litter. This deeper understanding of current attitudes toward smoke-free parks, especially among those who are receptive to partial restrictions, may offer insights into how best to inform the adoption, implementation, and sustainment of smoke-free park policies.

These findings suggest that the majority of U.S. adults support partial or complete restrictions on smoking in public parks, while opportunities exist to continue to build awareness of the harms of outdoor SHS exposure. Smoke-free policies covering settings where children live, work, learn and 
play-including parks—can reinforce tobacco-free norms as well as protect against SHS exposure. In contrast, permitting smoking in parks may undermine youth tobacco prevention efforts, because youth are particularly vulnerable to visual cues to smoke and to social norms [1]. As more localities embrace smoke-free policies in indoor public spaces, tobacco control stakeholders are expanding the frontier of tobacco-free environments to include public parks, as well as multiunit housing, public beaches, private vehicles, and college campuses $[1,6,7]$. Continued implementation of smoke-free park policies, in coordination with efforts to implement and enforce smoke-free policies in public indoor areas, could further expand the number of smoke-free environments frequented by children and improve the comprehensiveness of tobacco control efforts [1,7].

Our findings are subject to some limitations. First, these data are self-reported, and therefore, subject to social desirability bias, which could lead to overestimates of favorability. Second, these data are from 2009-2010; thus, the data may actually be underestimates that don't reflect more recent increases in favorable attitudes toward smoke-free parks. Third, the overall response rate for NATS was $37.6 \%$ and state-specific response rate ranged from $28.2 \%$ to $49.3 \%$, which could increase non-response bias; however, data were weighted for selection and non-response, so the impact of such bias on the reported estimates is likely minimal. Fourth, consistent with previous reports and recommendations for the 2009-2010 NATS, cellular telephone responses were excluded for states that had fewer than 200 cellular telephone respondents [33,37,38]; thus, state-specific estimates may be underrepresented for these states. Fifth, the NATS questionnaire provided limited detail about the types of parks or settings in parks for which the respondent favored smoke-free policies, such as outdoor sports stadiums and grounds, outdoor facility within hospitality venues, and playgrounds; therefore, it's not possible to ascertain whether respondents' favorability toward smoke-free policies may have varied based on specific locations within parks. Finally, limited sample size resulted in wide confidence intervals for some states, and for certain measures (e.g., non-combustible only tobacco use), estimates could not be presented because of statistical instability. However, all presented estimates had a relative standard error less than $30 \%$, which is a standard and generally recognized threshold used in the scientific literature to establish statistical stability of estimates from large population-based surveys $[36,38,39]$. Despite these limitations, the study has many strengths, including the use of a large, nationally representative sample and the ability to assess state-specific prevalence of favorable attitudes toward smoke-free parks among U.S. adults.

\section{Conclusions}

This study is the first to comprehensively assess the prevalence and correlates of favorable public attitudes toward smoke-free parks at the national level. The findings reveal that almost $40 \%$ of U.S. adults reported favorable attitudes towards completely smoke-free parks, and an additional $42 \%$ of adults reported attitudes supporting some level of smoking restrictions in parks. However, attitudes toward complete smoking restrictions in this setting vary by sociodemographic groups, current tobacco use status, and state. Efforts to educate the public about the dangers of SHS and the benefits of outdoor smoke-free environments could help reduce the extent of SHS exposure and social acceptability of tobacco use in parks.

Acknowledgments: No funding, direct or indirect, for CDC authors. Michelle Kegler funded by the National Cancer Institute under the State and Community Tobacco Control Initiative, Grant Number UO1-CA154282. The findings and conclusions in this report are those of the authors and do not necessarily represent the official position of the Centers for Disease Control and Prevention.

Author Contributions: Judy Kruger, Michelle Kegler, Brian King, Kristy Marynak designed the study. Amal Jama analyzed the data and all author helped interpret the data. Judy Kruger drafted the manuscript and all authors contributed to the revision and writing of the paper. All authors approved the final version of the manuscript to be submitted for consideration of publication.

Conflicts of Interest: The authors declare they have no competing interest to report. 


\section{Abbreviations}

The following abbreviations are used in this manuscript:

NATS National Adult Tobacco Survey

SHS Secondhand smoke

U.S. United States

\section{References}

1. United States Department of Health and Human Services. Preventing Tobacco Use among Young People: A Report of the Surgeon General; United States Department of Health and Human Services, Centers for Disease Control and Prevention: Atlanta, GA, USA, 1994.

2. United States Department of Health and Human Services. The Health Consequences of Involuntary Exposure to Tobacco Smoke: A Report of the Surgeon General; United States Department of Health and Human Services, Centers for Disease Control and Prevention: Atlanta, GA, USA, 2006.

3. United States Department of Health and Human Services. The Health Consequences of Smoking-50 Years of Progress: A Report of the Surgeon General; United States Department of Health and Human Services, Centers for Disease Control and Prevention: Atlanta, GA, USA, 2014.

4. Homa, D.M.; Neff, L.J.; King, B.A.; Caraballo, R.S.; Bunnell, R.E.; Babb, S.D.; Garrett, B.E.; Sosnoff, C.S.; Wang, L. Vital signs: Disparities in nonsmokers' exposure to secondhand smoke-United States, $1999-2012$. Morb. Mortal. Wkly. Rep. 2015, 64, 103-108.

5. World Health Organization. WHO Framework Convention on Tobacco Control; World Health Organization: Geneva, Switzerland, 2003.

6. Barnoya, J.; Navas-Acien, A. Protecting the world from secondhand tobacco smoke exposure: Where do we stand and where do we go from here? Nicotine Tob Res. 2013, 15, 789-804. [CrossRef] [PubMed]

7. Centers for Disease Control and Prevention. Best Practices for Comprehensive Tobacco Control Programs-2014; United States Department of Health and Human Services, Centers for Disease Control and Prevention, National Center for Chronic Disease Prevention and Health Promotion, Office on Smoking and Health: Atlanta, GA, USA, 2014.

8. King, B.; Mahoney, M.; Cummings, M.; Hyland, A. Intervention to promote smoke-free policies among multiunit housing operators. J. Public Health Manag. Pract. 2011, 17, E1-E8. [CrossRef] [PubMed]

9. Pizacani, B.; Laughter, D.; Menagh, K.; Stark, M.; Drach, L.; Hermann-Franzen, C. Moving multiunit housing providers toward adoption of smoke-free policies. Prev. Chronic Dis. 2011, 8, A21. [PubMed]

10. Hood, N.E.; Bernat, D.H.; Ferketich, A.K.; Danesh, D.; Klein, E.G. Community characteristics associated with smokefree park policies in the United States. Nicotine Tob. Res. 2014, 16, 828-835. [CrossRef] [PubMed]

11. Satterlund, T.D.; Cassady, D.; Treiber, J.; Lemp, C. Strategies implemented by 20 local tobacco control agencies to promote smoke-free recreation areas, California, 2004-2007. Prev. Chronic Dis. 2011, 8, A111. [PubMed]

12. Leung, R.; Mallya, G.; Dean, L.T.; Rizvi, A.; Dignam, L.; Schwarz, D.F. Instituting a smoke-free policy for city recreation centers and playgrounds, Philadelphia, Pennsylvania, 2010. Prev. Chronic Dis. 2013, 10 , E116. [CrossRef] [PubMed]

13. Lopez, M.J.; Fernandez, E.; Gorini, G.; Moshammer, H.; Polanska, K.; Clancy, L.; Dautzenberg, B.; Delrieu, A.; Invernizzi, G.; Muñoz, G.; et al. Exposure to secondhand smoke in terraces and other outdoor areas of hospitality venues in eight European countries. PLoS ONE 2012, 7. [CrossRef] [PubMed]

14. Johns, M.; Coady, M.; Chan, C.; Farley, S.M.; Kansagra, S.M. Evaluating New York City's smoke-free parks and beaches law: A critical multiple list approach to assessing behavioral impact. Am. J. Community Psychol. 2013, 51, 254-263. [CrossRef] [PubMed]

15. Thomson, G.; Wilson, N.; Edwards, R. At the frontier of tobacco control: A brief review of public attitudes toward smoke-free outdoor places. Nicotine Tob. Res. 2009, 11, 584-590. [CrossRef] [PubMed]

16. Americans Nonsmokers' Rights Foundation. Municipalities with Smokefree Park Laws, Enacted as of 2 October 2015. Available online: http://no-smoke.org/pdf/SmokefreeParks.pdf (accessed on 25 April 2016).

17. Waddell, E.N.; Farley, S.N.; Mandel-Ricci, J.; Kansagra, S.M. Public support for smoke-free air strategies among smokers and nonsmokers, New York City, 2010-2012. Prev. Chronic Dis. 2014, 11, 130263. [CrossRef] [PubMed] 
18. Bayer, R.; Bachysski, K.E. Banning smoking in parks and on beaches: Science, policy and the politics of denormalization. Health Affairs 2013, 32, 1291-1298. [CrossRef] [PubMed]

19. Johns, M.; Farley, S.M.; Rajulu, D.T.; Kansagra, S.M.; Juster, H.R. Smoke-free parks and beaches: An interrupted time-series study of behavioural impact in New York City. Tob. Control 2015, 24, 497-500. [CrossRef] [PubMed]

20. Alesci, N.; Forster, J.; Blaine, T. Smoking visibility, perceived acceptability, and frequency in various locations among youth and adults. Prev. Med. 2003, 36, 272-281. [CrossRef]

21. Sureda, X.; Fenandez, E.; Lopez, M.J.; Nebot, M. Secondhand tobacco smoke exposure in open and semi-open stings: A systematic review. Environ. Health Perspect. 2013, 121, 766-773. [CrossRef] [PubMed]

22. Kaufman, P.; Zhang, B.; Bondy, S.J.; Klepeis, N.; Ferrence, R. Not just "a few wisps": Real-time measurement of tobacco smoke at entrances to office buildings. Tob. Control 2011, 20, 212-218. [CrossRef] [PubMed]

23. Klepeis, N.; Ott, W.; Switzer, P. Real-time measurement of outdoor tobacco smoke particles. J. Air Waste Manag. Assoc. 2007, 57, 522-534. [CrossRef] [PubMed]

24. Hall, J.C.; Bernert, J.T.; Hall, D.B.; St. Helen, G.; Kudon, L.H.; Naeher, L.P. Assessment of exposure to secondhand smoke at outdoor bars and family restaurants in Athens, Georgia, using salivary cotinine. J. Occup. Environ. Hyg. 2009, 6, 698-704. [CrossRef] [PubMed]

25. Licht, A.S.; Hyland, A.; Travers, M.J.; Chapman, S. Secondhand smoke exposure levels in outdoor hospitality venues: A qualitative and quantitative review of the research literature. Tob. Control. 2013, 22, 172-179. [CrossRef] [PubMed]

26. International Agency for Research on Cancer (IARC). Volume 13. Evaluating the Effectiveness of Smoke-Free Policies; IARC: Lyon, France, 2009.

27. McMillen, R.C.; Winickoff, J.P.; Klein, J.D.; Weitzman, M. U.S. adult attitude and practices regarding smoking restrictions and child exposure to environmental tobacco smoking: Changes in the social climate from 2000-2001. Pediatrics 2003, 112, e55-e60. [CrossRef] [PubMed]

28. Stevenson, A.R.; Bradshaw, R.; Cook, J.; Cunningham, R.; Riddick, L.; Miller, R.; Edwards, R.; Wilson, N.; Thomson, G. Majority of smokers and non-smokers in favour of smokefree parks in New Zealand. N. Z. Med. J. 2008, 121, 108-110. [PubMed]

29. Klein, E.G.; Bernat, D.H.; Forster, J.L. Young adult perceptions of smoking in outdoor park areas. Health Place 2012, 18, 1042-1045. [CrossRef] [PubMed]

30. Kaufman, P.; Griffin, K.; Cohen, J.; Perkins, N.; Ferrence, R. Smoking in urban outdoor public places: Behavior, experiences, and implications for public health. Health Place 2012, 16, 961-968. [CrossRef] [PubMed]

31. Novotny, T.E.; Hardin, S.N.; Hovda, L.R.; Novotny, D.J.; McLean, M.K.; Khan, S. Tobacco and cigarette butt consumption in humans and animals. Tob. Control 2011, 20, i17-i20. [CrossRef] [PubMed]

32. Centers for Disease Control and Prevention. National Adult Tobacco Survey 2014. Available online: http://www.cdc.gov/tobacco/data_statistics/surveys/nats/ (accessed on 25 April 2016).

33. Centers for Disease Control and Prevention, Office on Smoking and Health and ICF. 2009-2010 National Adult Tobacco Survey Methodology Report; Centers for Disease Control and Prevention, Office on Smoking and Health and ICF: Atlanta, GA, USA, 2011.

34. Council of American Survey and Research Organizations (CASRO). Code of Standards and Ethics for Survey Research. 2011. Available online: https://c.ymcdn.com/sites/www.casro.org/resource/resmgr/casro_ code_of_standards.pdf (accessed on 25 April 2016).

35. King, B.A.; Patel, R.; Nguyen, K.H.; Dube, S.R. Trends in awareness and use of electronic cigarettes among U.S. adults, 2010-2013. Nicotine Tob. Res. 2015, 17, 219-227. [CrossRef] [PubMed]

36. Americans Nonsmokers' Rights Foundation. U.S. Tobacco Control Laws Database. 2015. Available online: http:/ / www.no-smoke.org/document.php?id=313 (accessed on 25 April 2016).

37. Kruger, J.; Patel, R.; Kegler, M.; Babb, S.D.; King, B.A. Perceptions of harm from secondhand smoke exposure among U.S. adults, 2009-2010. Tob. Induc. Dis. 2016, 14, 3. [CrossRef] [PubMed]

38. King, B.A.; Dube, S.R.; Tynan, M.A. Attitudes toward smoke-free workplaces, restaurants, and bars, casinos, and clubs among U.S. adults: Findings from the 2009-2010 National Adult Tobacco Survey. Nicotine Tob. Res. 2013, 15, 1464-1470. [CrossRef] [PubMed]

39. Pacheco, J. Attitudinal policy feedback and public opinion: The impact of smoking bans on attitudes towards smokers, secondhand smoke, and antismoking policies. Public Opin Q. 2013. [CrossRef] 
40. Thomson, G.; Wilson, N.; Edwards, R.; Woodward, A. Should smoking outside public spaces be banned? BMJ 2008, 337, a2806. [CrossRef] [PubMed]

41. Hyslop, B.; Thomson, G. Smokefree outdoor areas without the smoke-police: The New Zealand local authority experience. N. Z. Med. J. 2009, 122, 67-79. [PubMed]

42. Agaku, I.T.; King, B.A.; Husten, C.G.; Bunnell, R.; Ambrose, B.K.; Hu, S.S.; Holder-Hayes, E.; Day, H.R.; Centers for Disease Control and Prevention (CDC). Tobacco product use among adults-United States 2012-2013. Morb. Mortal. Wkly. Rep. 2014, 63, 542-547.

43. Francis, K.A.; Abramsohn, E.M.; Park, H.Y. Policy-driven tobacco control. Tob. Control 2010, 19, i16-i20. [CrossRef] [PubMed]

(C) 2016 by the authors; licensee MDPI, Basel, Switzerland. This article is an open access article distributed under the terms and conditions of the Creative Commons Attribution (CC-BY) license (http:/ / creativecommons.org/licenses/by/4.0/). 